\title{
Interacting resonant-level model with long-range interactions: Fast screening and suppression of the zero-bias conductance
}

\author{
E. Perfetto, ${ }^{1}$ G. Stefanucci, ${ }^{1,2,3}$ and M. Cini ${ }^{1,3}$ \\ ${ }^{1}$ Dipartimento di Fisica, Universitá di Roma Tor Vergata, Via della Ricerca Scientifica 1, I-00133 Rome, Italy \\ ${ }^{2}$ European Theoretical Spectroscopy Facility (ETSF) \\ ${ }^{3}$ Istituto Nazionale di Fisica Nucleare, Laboratori Nazionali di Frascati, Via E. Fermi 40, I-00044 Frascati, Italy
}

(Received 3 October 2011; revised manuscript received 9 March 2012; published 19 April 2012)

\begin{abstract}
The effects of long-range interactions in quantum transport are still largely unexplored, mainly due to the difficulty of devising efficient embedding schemes. In this work we present a substantial progress in the interacting resonant level model by reducing the problem to the solution of Kadanoff-Baym-like equations with a correlated embedding self-energy. The method allows us to deal with short- and long-range interactions and is applicable from the transient to the steady-state regime. Furthermore, memory effects are consistently incorporated and the results are not plagued by negative densities or nonconservation of the electric charge. We employ the method to calculate densities and currents with long-range interactions appropriate to low-dimensional leads, and show the occurrence of a jamming effect, which drastically reduces the screening time and suppresses the zero-bias conductance. None of these effects are captured by short-range model interactions.
\end{abstract}

DOI: 10.1103/PhysRevB.85.165437

PACS number(s): 73.63.-b, 05.60.Gg, 71.10.Pm

\section{INTRODUCTION}

Electron correlations have profound implications on the transport properties of nanoscale devices. ${ }^{1}$ Local interactions within small molecules or quantum dots contacted to leads give rise to peculiar phenomena like the Kondo effect ${ }^{2}$ and Coulomb blockade, ${ }^{3}$ and have been the subject of several studies. Much less attention has been devoted instead to the nonlocal interactions responsible for interfacial screening and polarization-induced renormalizations of the molecular levels. Recently, a short-range (SR) dot-lead interaction has been shown to reduce the quasiparticle gap in equilibrium ${ }^{4-7}$ and to reopen it at sufficiently large biases. ${ }^{8}$ In the interacting resonant level model (IRLM) the SR interaction is also at the origin of a negative differential conductance with an interaction-dependent power-law decay ${ }^{9-12}$ as well as of an overall enhancement of the off-resonance conductance. ${ }^{13,14}$

In recent years the experimental progress in producing low-dimensional conducting wires has been accompanied by an increasing number of theoretical studies on the IRLM with single-channel leads. In these systems, however, screening occurs on length scales much longer than a few Angstrom and long-range (LR) interactions should be more appropriate for a realistic description. Unfortunately, the techniques developed to deal with SR interactions are not directly exportable to study LR ones. ${ }^{15}$ The difficulty stems from the impracticability of combining many-body methods with embedding techniques, hence reducing the problem to the evaluation of the Green's function of a finite and interacting open system. ${ }^{16,17}$ Recently Elste and coworkers ${ }^{18}$ approached the problem using the rate equations (REs) method in the IRLM with Luttinger liquid leads. The REs, however, are not reliable in the transient regime and underestimate the steady-state polarizability of the dot, as we will clearly show below. The fundamental questions which remain at present totally unanswered are therefore: What is the impact of a LR dot-lead interaction on the $I-V$ curve? How does the screening time change from SR to LR interactions?
In this work we consider the IRLM as the prototype model to address the above issues. We study the real-time evolution of the current and dot-density after the sudden switch-on of a bias voltage for both SR and LR interactions. Our results indicate that LR interactions produce a jamming effect in the leads that (i) shortens the screening time and (ii) drastically suppresses the zero-bias conductance.

The proposed methodology to conclude (i) and (ii) is based on a truncation of the equations of motion for dressed correlators. The procedure leads to Kadanoff-Baym-like equations with a correlated embedding self-energy, which incorporates all interaction and memory effects. Our approach overcomes the negative probability problem ${ }^{19}$ of the RE and is, at the same time, charge conserving. The final equations are exact in the uncontacted case as well as in the noninteracting case and several analytic results are obtained in the steadystate, including a Meir-Wingreen-like formula for the current. We benchmarked this formula against recent results with SR interaction obtained using field theoretical methods, ${ }^{9}$ DMRG,${ }^{9,14}$ and other renormalization group approaches, ${ }^{11-13}$ and found the same qualitative behavior.

The plan of the paper is as follows. In Sec. II we introduce the IRLM for an arbitrary dot-lead interaction and employ the bosonization method followed by a Lang-Firsov transformation to formally eliminate the dot-lead coupling. This allows us to set up a suitable truncation scheme and derive a set of Kadanoff-Baym equations with a correlated embedding self-energy. In Sec. III we compare these equations to the well-known rate equations and show that the proposed method is superior to describe both the transient and the steady-state regimes. We further show that our approximation is in very good agreement with the exact solution for shortrange interactions. Encouraged by these results we extend the analysis to long-range interactions and discover a remarkable effect, namely the suppression of the zero-bias conductance. A summary of the main findings and concluding remarks are made in Sec. IV. 


\section{KADANOFF-BAYM EQUATIONS FOR THE INTERACTING RESONANT LEVEL MODEL}

We consider the IRLM described by the Hamiltonian

$$
\begin{aligned}
\hat{H} & =-\sum_{\alpha} i \alpha v \int d x \hat{\psi}_{\alpha}^{\dagger}(x) \partial_{x} \hat{\psi}_{\alpha}(x)+\varepsilon_{d} \hat{n}_{d} \\
& +\int d x U(x) \hat{\rho}(x) \hat{n}_{d}+\sum_{\alpha}\left[T_{\alpha}^{*} \hat{\psi}_{\alpha}^{\dagger}(0) \hat{d}+\text { H.c. }\right],
\end{aligned}
$$

with $\alpha= \pm 1$ for $R$ and $L$ electrons moving with velocity $v$, $\hat{n}_{d}=\hat{d}^{\dagger} \hat{d}$, and $\hat{\rho}=\sum_{\alpha} \hat{\rho}_{\alpha}=\sum_{\alpha} \hat{\psi}_{\alpha}^{\dagger} \hat{\psi}_{\alpha}$. In Eq. (1) $\varepsilon_{d}$ is the dot energy (gate voltage), $T_{\alpha}$ is the tunneling amplitude, and the dot-lead Coulomb interaction $U(x)$ can be either SR or LR. The system is driven out of equilibrium by the bias perturbation

$$
\hat{H}_{B}=\sum_{\alpha} V_{\alpha} \int d x \hat{\rho}_{\alpha}(x)=\sum_{\alpha} V_{\alpha} \hat{N}_{\alpha}
$$

with $\hat{N}_{\alpha}$ the number of electrons with chirality $\alpha$. For simplicity we consider a single-level dot but the derivation below remains applicable to multilevel dots by replacing scalar Green's functions with matrices. For a nonperturbative treatment of the interaction we bosonize the fermion operators ${ }^{20,21}$

$$
\hat{\psi}_{\alpha}(x)=\frac{\eta_{\alpha}}{\sqrt{2 \pi a}} e^{-2 \sqrt{\pi} i \alpha \hat{\phi}_{\alpha}(x)},
$$

with boson field

$$
\hat{\phi}_{\alpha}(x)=i \alpha \sum_{q>0} \Lambda_{q}\left(\hat{b}_{\alpha q}^{\dagger} e^{-i \alpha q x}-\text { H.c. }\right)-\frac{\sqrt{\pi} x \hat{N}_{\alpha}}{\mathcal{L}}
$$

and $\eta_{\alpha}$ the anticommuting Klein factor. In the mode expansion of the boson field $\Lambda_{q}=\frac{e^{-\frac{a q}{2}}}{\sqrt{2 \mathcal{L} q}}$, with $\mathcal{L}$ the length of the system and $a$ a short-distance cutoff. The bosonized form of the electron density takes the form

$$
\begin{aligned}
\hat{\rho}_{\alpha}(x) & =-\partial_{x} \hat{\phi}_{\alpha}(x) / \sqrt{\pi} \\
& =\sum_{q>0} \Lambda_{q} q\left(\hat{b}_{\alpha q}^{\dagger} e^{-i \alpha q x}-\text { H.c. }\right)+\frac{\hat{N}_{\alpha}}{\mathcal{L}},
\end{aligned}
$$

and hence the bosonized Hamiltonian reads

$$
\begin{aligned}
\hat{H}= & \sum_{\alpha, q>0} v q \hat{b}_{\alpha q}^{\dagger} \hat{b}_{\alpha q}+\varepsilon_{d} \hat{n}_{d} \\
& -\sum_{\alpha, q>0} \frac{\Lambda_{q} q}{\sqrt{\pi}} U_{q}\left(\hat{b}_{\alpha q}^{\dagger}+\hat{b}_{\alpha q}\right) \hat{n}_{d}+U_{0} \sum_{\alpha} \frac{\hat{N}_{\alpha}}{\mathcal{L}} \hat{n}_{d} \\
& +\sum_{\alpha}\left[\frac{T_{\alpha}^{*} \eta_{\alpha}^{\dagger}}{\sqrt{2 \pi a}} \exp \left(-2 \sqrt{\pi} \sum_{q>0} \Lambda_{q}\left(\hat{b}_{\alpha q}^{\dagger}-\hat{b}_{\alpha q}\right)\right) \hat{d}+\text { H.c. }\right],
\end{aligned}
$$

where $U_{q}=\int d x e^{i q x} U(x)$ and we used $U(x)=U(-x)$. Next we perform a Lang-Firsov transformation to (formally) eliminate the dot-lead coupling. The unitary operator

$$
\hat{\mathcal{U}}=\exp \left(2 \sqrt{\pi} \sum_{\alpha q} \frac{\Lambda_{q}}{2 \pi v} U_{q}\left(\hat{b}_{\alpha q}^{\dagger}-\hat{b}_{\alpha q}\right) \hat{n}_{d}\right)
$$

transforms the original Hamiltonian into $\hat{H}^{\prime}=\hat{\mathcal{U}}^{\dagger} \hat{H} \hat{\mathcal{U}}$ with (from now on all sums are over $q>0$ )

$$
\begin{aligned}
\hat{H}^{\prime}= & \sum_{\alpha q} v q \hat{b}_{\alpha q}^{\dagger} \hat{b}_{\alpha q}+\tilde{\varepsilon}_{d} \hat{n}_{d}+U_{0} \sum_{\alpha} \frac{\hat{N}_{\alpha}}{\mathcal{L}} \hat{n}_{d} \\
& +\sum_{\alpha}\left[T_{\alpha}^{*} \hat{f}_{\alpha 0}^{\dagger} \hat{d}+\text { H.c. }\right] .
\end{aligned}
$$

In the transformed Hamiltonian it appears the renormalized fermion field

$$
\hat{f}_{\alpha x}=\frac{\eta_{\alpha}}{\sqrt{2 \pi a}} \exp \left(2 \sqrt{\pi} \sum_{\beta q} \Lambda_{q} W_{\alpha \beta q}\left(\hat{b}_{\beta q}^{\dagger} e^{-i \alpha q x}-\hat{b}_{\beta q} e^{i \alpha q x}\right)\right),
$$

evaluated in $x=0$, with the effective interactions $W_{R R q}=$ $W_{L L q}=1+U_{q} /(2 \pi v)$ and $W_{R L q}=W_{L R q}=U_{q} /(2 \pi v)$, and the renormalized energy level $\tilde{\varepsilon}_{d}=\varepsilon_{d}+\sum_{q} \frac{e^{-a q}}{\pi v \mathcal{L}}\left|U_{q}\right|^{2}$. In the new basis the ground state of the isolated leads (i.e., with $T_{\alpha}=$ 0 ) is the vacuum $|0\rangle$ of the boson operators $\hat{b}_{\alpha q}$. We can exploit this property to build the proper initial conditions by time propagation. We will consider the system initially uncontacted $\left(T_{\alpha}=0\right)$, then switch on the contacts at time $t=0$ and let the current and dot-density relax. After relaxation, say at time $t_{0}$, we will bias the leads and study the screening dynamics from the transient to the steady state. This procedure simulates with high accuracy the so-called partition-free scheme, ${ }^{22,23}$ as demonstrated in Refs. 24-26.

We define the dot Green's function on the Keldysh contour as

$$
G\left(z, z^{\prime}\right)=\frac{1}{i}\left\langle\mathcal{T}\left\{\hat{d}(z) \hat{d}^{\dagger}\left(z^{\prime}\right)\right\}\right\rangle,
$$

where $\mathcal{T}$ is the contour ordering, operators are in the Heisenberg picture with respect to $\hat{H}^{\prime}+\hat{H}_{B}$ (the bias perturbation does not change after the transformation), and the average is taken over the uncontacted ground state $|0\rangle \otimes|n\rangle,|n\rangle$ being the state of the dot with single $(n=1)$ or zero $(n=0)$ occupancy. The Green's function obeys the equation of motion (EOM)

$$
\left(i \partial_{z}-\tilde{\varepsilon}_{d}\right) G\left(z, z^{\prime}\right)=\delta\left(z, z^{\prime}\right)+\sum_{\alpha} T_{\alpha}(z) G_{\alpha 0}\left(z, z^{\prime}\right),
$$

where

$$
G_{\alpha x}\left(z, z^{\prime}\right)=\frac{1}{i}\left\langle\mathcal{T}\left\{\hat{f}_{\alpha x}(z) \hat{d}^{\dagger}\left(z^{\prime}\right)\right\}\right\rangle
$$

is the dot-lead Green's function. ${ }^{27}$ To close the EOM we derive $G_{\alpha x}$ with respect to its first argument and find

$$
\begin{aligned}
& \left(i \partial_{z}+i \alpha v \partial_{x}-V_{\alpha}(z)\right) G_{\alpha x}\left(z, z^{\prime}\right) \\
& \quad=\frac{1}{i} \sum_{\beta}\left\langle\mathcal{T}\left\{\left[T_{\beta}^{*} \hat{f}_{\beta 0}^{\dagger} \hat{d}+\text { H.c., } \hat{f}_{\alpha x}\right](z) \hat{d}^{\dagger}(z)\right\}\right\rangle .
\end{aligned}
$$

The computation of the correlator in the right-hand side of Eq. (13) is a formidable task. In order to proceed we approximate it by $T_{\alpha}^{*}\left\langle\left(\hat{f}_{\alpha 0}^{\dagger} \hat{f}_{\alpha x}+\hat{f}_{\alpha x} \hat{f}_{\alpha 0}^{\dagger}\right)(z)\right\rangle_{P} G\left(z, z^{\prime}\right)$, where $\langle\cdots\rangle_{P}$ signifies that operators are in the Heisenberg picture with respect to the uncontacted Hamiltonian. This approximation is at the basis of our truncation scheme and corresponds to discard virtual tunneling processes between two consecutive interactions of the propagating electron. It becomes exact in 
the noninteracting case as well as in the uncontacted case. Our approximation remains very accurate also for small $T_{\alpha}$ since it correctly reproduces recent results on the IRLM with SR interaction (see below).

To solve the EOM for $G_{\alpha x}$ we define

$$
g_{\alpha x \alpha x^{\prime}}\left(z, z^{\prime}\right)=\frac{1}{i}\left\langle\mathcal{T}\left\{\hat{f}_{\alpha x}(z) \hat{f}_{\alpha x^{\prime}}^{\dagger}\left(z^{\prime}\right)\right\}\right\rangle_{P},
$$

which satisfies the EOM

$$
\begin{aligned}
& \left(i \partial_{z}+i \alpha v \partial_{x}-V_{\alpha}(z)\right) g_{\alpha x \alpha x^{\prime}}\left(z, z^{\prime}\right) \\
& \quad=\delta\left(z, z^{\prime}\right)\left\langle\left(\hat{f}_{\alpha x} \hat{f}_{\alpha x^{\prime}}^{\dagger}+\hat{f}_{\alpha x^{\prime}}^{\dagger} \hat{f}_{\alpha x}\right)(z)\right\rangle_{P} .
\end{aligned}
$$

We can now perform a standard embedding and write the dot Green's function as the solution of

$$
\left(i \partial_{z}-\tilde{\varepsilon}_{d}\right) G\left(z, z^{\prime}\right)-\int_{\gamma} d \bar{z} \sum_{\alpha} \Sigma_{\alpha}(z, \bar{z}) G\left(\bar{z}, z^{\prime}\right)=\delta\left(z, z^{\prime}\right),
$$

where $\Sigma_{\alpha}\left(z, z^{\prime}\right)=\left|T_{\alpha}\right|^{2} g_{\alpha 0 \alpha 0}\left(z, z^{\prime}\right)$ is the correlated embedding self-energy and the integral runs over the Keldysh contour. Using the Langreth rules ${ }^{28}$ Eq. (16) is converted into a coupled system of Kadanoff-Baym equations ${ }^{29-32}$ (KBEs), which we solve numerically. The real-time Keldysh components of $\Sigma$ can be evaluated exactly using the bosonization method ${ }^{20,21}$ and read

$$
\Sigma_{\alpha}^{\lessgtr}\left(t, t^{\prime}\right)= \pm \frac{i\left|T_{\alpha}\right|^{2}}{2 \pi a} e^{-i \varphi_{\alpha}(t)} e^{Q\left[ \pm\left(t-t^{\prime}\right)\right]} e^{i \varphi_{\alpha}\left(t^{\prime}\right)},
$$

with phase $\varphi_{\alpha}(t)=\int_{0}^{t} d \bar{t} V_{\alpha}(\bar{t})$ and interaction dependent exponent

$Q(t)=\sum_{q} \frac{2 \pi}{\mathcal{L} q} e^{-a q}\left(e^{i v q t}-1\right)\left[1-\frac{U_{q}}{\pi v}+\frac{1}{2}\left(\frac{U_{q}}{\pi v}\right)^{2}\right]$.

From solution of Eq. (16) we can easily calculate the dotdensity from $\left\langle\hat{n}_{d}(t)\right\rangle=-i G^{<}(t, t)$. Similarly, the current $I_{\alpha}$ at the interface between the dot and lead $\alpha$ can be calculate from

$$
\begin{aligned}
I_{\alpha}(z) & =\partial_{z}\left\langle\hat{N}_{\alpha}(z)\right\rangle=-i T_{\alpha}^{*}\left\langle\hat{f}_{\alpha 0}^{\dagger}(z) \hat{d}(z)\right\rangle+\text { H.c. } \\
& =\int_{\gamma} d \bar{z} \Sigma_{\alpha}(z, \bar{z}) G(\bar{z}, z)+\text { H.c. }
\end{aligned}
$$

In the steady-state regime $G\left(t, t^{\prime}\right)$ depends only on the time difference $t-t^{\prime}$ and the current $\bar{I}=I_{L}(t \rightarrow \infty)=-I_{R}(t \rightarrow$ $\infty)$ is given by a Meir-Wingreen-like formula

$$
\bar{I}=\int \frac{d \omega}{2 \pi} \frac{\Sigma_{L}^{>}(\omega) \Sigma_{R}^{<}(\omega)-\Sigma_{L}^{<}(\omega) \Sigma_{R}^{>}(\omega)}{\left|\omega-\tilde{\varepsilon}_{d}-\sum_{\alpha} \Sigma_{\alpha}^{\mathrm{R}}(\omega)\right|^{2}} .
$$

Remarkably, the current cannot be written in terms of the difference between the leads' Fermi functions despite the left and right contacts are the same (proportionate coupling). ${ }^{16}$

\section{RESULTS}

\section{A. Comparison with the rate equations}

Our analysis starts by comparing the present approximation to the RE method, recently employed in a similar context. ${ }^{18}$ It is worth recalling that within the RE method only the equal-time

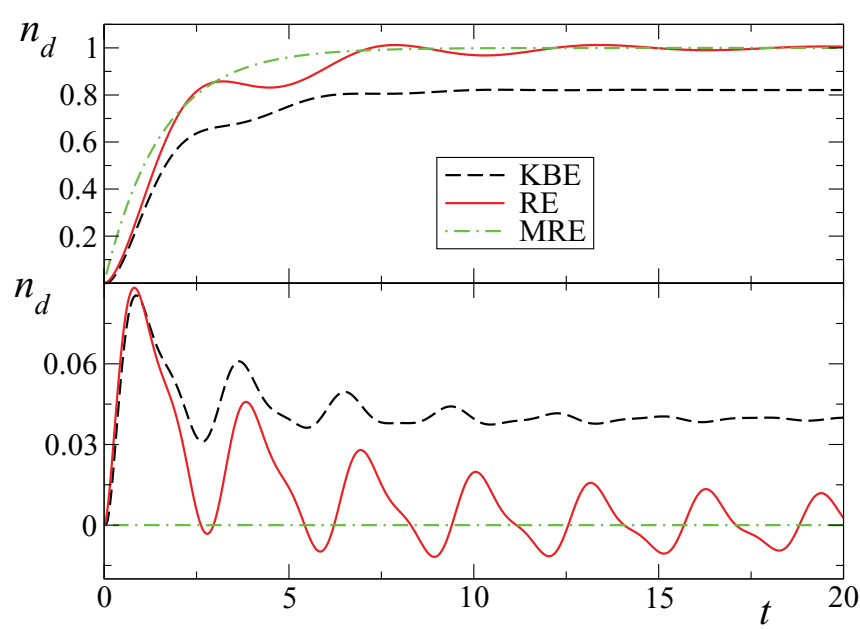

FIG. 1. (Color online) Time-dependent density for SR intraction using KBEs (dashed), REs (solid), and MREs (dotted) for dot energy $\tilde{\varepsilon}_{d}=0$ (top panel) and $\tilde{\varepsilon}_{d}=3$ (bottom panel) and initial dot density $n=0$. The remaining parameters are $V_{L}=V_{R}=1, \gamma=0.2, U=$ $0.5, t_{0}=0$ (partitioned scheme). Units are $10^{-1} v / a$ for $V_{\alpha}, \gamma, \tilde{\varepsilon}_{d}$, $10 a / v$ for $t$ and $2 \pi v$ for $U$.

density matrix is accessible. In the non-Markovian case the RE to be solved reads

$$
\begin{aligned}
i \partial_{t} G^{<}(t, t)= & i \sum_{\alpha} \int_{0}^{t} d \bar{t} 2 \operatorname{Re}\left[-i \Sigma_{\alpha}^{R}(t, \bar{t}) e^{i \tilde{\varepsilon}_{d}(t-\bar{t})}\right] G^{<}(\bar{t}, \bar{t}) \\
& +\sum_{\alpha} \int_{0}^{t} d \bar{t} 2 \operatorname{Re}\left[i \Sigma_{\alpha}^{<}(t, \bar{t}) e^{i \tilde{\varepsilon}_{d}(t-\bar{t})}\right]
\end{aligned}
$$

which in the Markovian limit reduces to

$$
i \partial_{t} G^{<}(t, t)=i\left(P_{0 \rightarrow 1}+P_{1 \rightarrow 0}\right) G^{<}(t, t)-P_{0 \rightarrow 1},
$$

with constant tunneling rates

$$
\begin{aligned}
P_{0 \rightarrow 1} & =\sum_{\alpha} \int_{0}^{\infty} d t 2 \operatorname{Re}\left[-i \Sigma_{\alpha}^{<}(t, 0) e^{i \tilde{\varepsilon}_{d} t}\right], \\
P_{1 \rightarrow 0} & =\sum_{\alpha} \int_{0}^{\infty} d t 2 \operatorname{Re}\left[i \Sigma_{\alpha}^{>}(t, 0) e^{i \tilde{\varepsilon}_{d} t}\right],
\end{aligned}
$$

denoting the probability of absorption (emission) of one electron in (from) the dot, respectively.

In Fig. 1 we plot the time-dependent dot density using the KBEs, the REs and their Markovian version (MREs) for a SR interaction $U_{q}=U$. Both the $\mathrm{KBE}$ and $\mathrm{RE}$ densities exhibit oscillations with frequencies associated to chargeneutral excitations. As anticipated, however, the REs suffer from the negative-density problem ${ }^{19}$ (bottom panel). The MRE density is instead always non-negative but the lack of memory washes out the oscillations and the transient becomes a featureless exponential (top panel). The KBE density is superior also at the steady state. Both the REs and MREs predict a zero-temperature steady-state density either 0 or 1 and hence severely underestimate the dot polarizability. We also verified that the $\mathrm{KBE}$ approach is charge conserving since it fulfills with high numerical accuracy the continuity equation $d\left\langle\hat{n}_{d}(t)\right\rangle / d t=I_{R}(t)+I_{L}(t)$ at every time (not shown). 


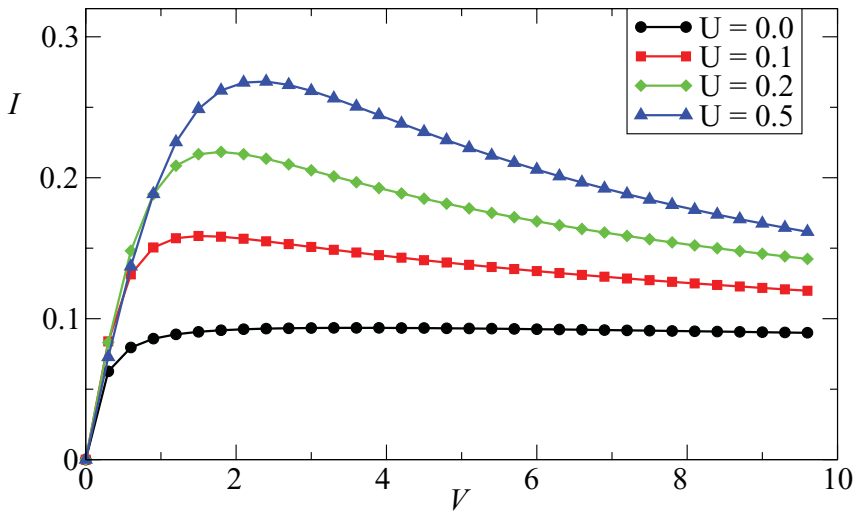

FIG. 2. (Color online) $I-V$ curve for SR interaction. The parameters are $\gamma=0.2, \tilde{\varepsilon}_{d}=0$. Units are $10^{-2} v / a$ for $I, V, \gamma, \tilde{\varepsilon}_{d}$, and $2 \pi v$ for $U$.

\section{B. Short-range interaction}

We next calculate the steady-state current for a SR interaction and in the symmetric case $\left(\tilde{\varepsilon}_{d}=0, T_{L}=T_{R}=T, V_{L}=\right.$ $-V_{R}=V>0$ ) recently considered by several authors. ${ }^{9,11,12}$ The $I-V$ curves resulting from Eq. (20) are shown in Fig. 2. The integral (20) can be accurately approximated by evaluating all $\Sigma$ in $\omega=0,{ }^{33}$ thus yielding the following analytic result

$$
\bar{I}_{\mathrm{SR}}(V) \simeq\left(\frac{V a}{v}\right)^{\beta-1} \frac{\gamma}{\pi \Gamma(\beta)} \tan ^{-1}\left[\frac{V}{\gamma}\left(\frac{V a}{v}\right)^{1-\beta} \Gamma(\beta)\right],
$$

with exponent $\beta(U)=1+\frac{U(U-2 \pi v)}{2 \pi^{2} v^{2}}$ and tunneling rate $\gamma=$ $|T|^{2} / v$. The above expression is in excellent agreement with the exact results of Ref. 9. In particular it reproduces the universal Ohmic behavior $\bar{I}_{\mathrm{SR}}(V) \simeq V / \pi$ at small bias ${ }^{34}$ (with $\sigma_{0}=1 / 2 \pi$ the quantum of conductance), and the negative differential conductance with the nonuniversal power-law decay $\bar{I}_{\mathrm{SR}}(V) \sim V^{\beta-1}$ at large bias (the REs fail again here). Note that the exponent $\beta$ is exactly that of Ref. 9. Equation (24) enjoys the self-duality $U \rightarrow 2 \pi v-U$ which, as discussed in Ref. 9, is the continuum version of the lattice self-duality $U \rightarrow 1 / U$. Furthermore at the self-dual point $U=\pi v$, the $I-V$ curves of Eq. (24) for different $\gamma$ collapse on one single curve if we rescale $V \rightarrow \gamma^{3 / 2} V$ and $I \rightarrow \gamma^{3 / 2} I$. This rescaling is again in agreement with Ref. 9, which found the same result by a best fitting.

\section{Long-range interaction}

We can now present the most important numerical results of the paper, i.e., the current with LR interaction $U_{q}=$ $-W \ln (a q)^{2}$ (corresponding to the soft-Coulomb interaction $U(x)=W / \sqrt{x^{2}+a^{2}}$ in real space). In this case the function $Q(t)$ as well as the integral in Eq. (20) must be evaluated numerically. In Fig. 3 we display the $I-V$ curve for several $W$. The behavior is qualitatively different from the SR case. In particular the zero-bias conductance is strongly suppressed with increasing $W$. Due to the LR nature of the interaction the addition (removal) of an electron to (from) the dot induces a charge depletion (accumulation), which extends smoothly deep inside the leads (jamming effect). For a current to

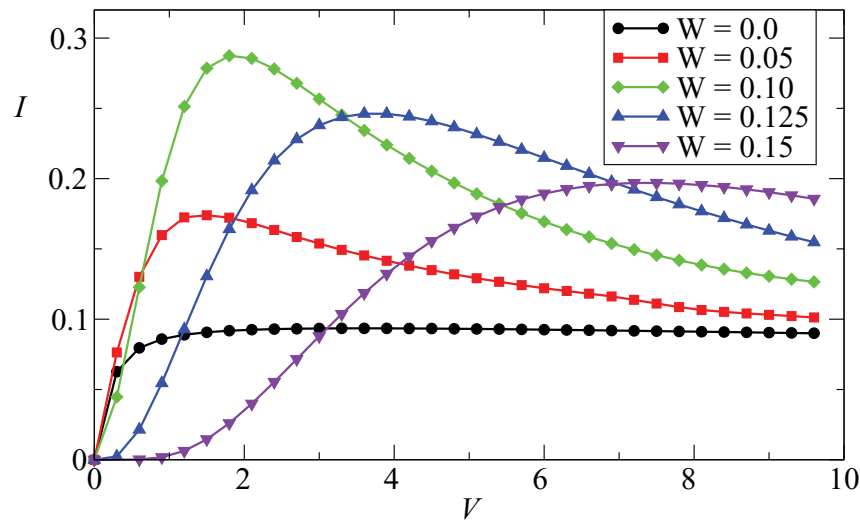

FIG. 3. (Color online) $I-V$ curve for LR interaction. Same parameters and units as in Fig. 2. $W$ is in units of $2 \pi v$.

flow the bias must be larger than the polarization energy of this particle-hole collective state. This picture also explains a common feature of the SR and LR $I-V$ curves (i.e., the existence of an optimal value of the interaction strength for which the current has a maximum at fixed bias). Increasing the interaction from zero the electron density diminishes close to the dot, thus enhancing the effective tunneling rate (Coulomb deblocking). However, increasing the interaction further the particle-hole binding energy becomes larger than the charge-transfer energy $V_{L}-V_{R}$ to move an electron from one lead to the other, and the current starts decreasing.

In Fig. 4 we show the cross-over from short- to longrange interactions using a soft-Yukawa-type of interaction $U(x)=W e^{-|x| / \lambda} / \sqrt{x^{2}+a^{2}}$, with varying screening length $\lambda$. We observe that our prediction on the suppression of the conductance is quite robust and is not an artifact of the regularization procedure. This prediction could be experimentally verified in, e.g., a quantum dot coupled via tunneling barriers to single-channel quantum wires or to the edge states of two-dimensional (2D) quantum Hall liquids. This experimental setup was proposed in Ref. 35 but the

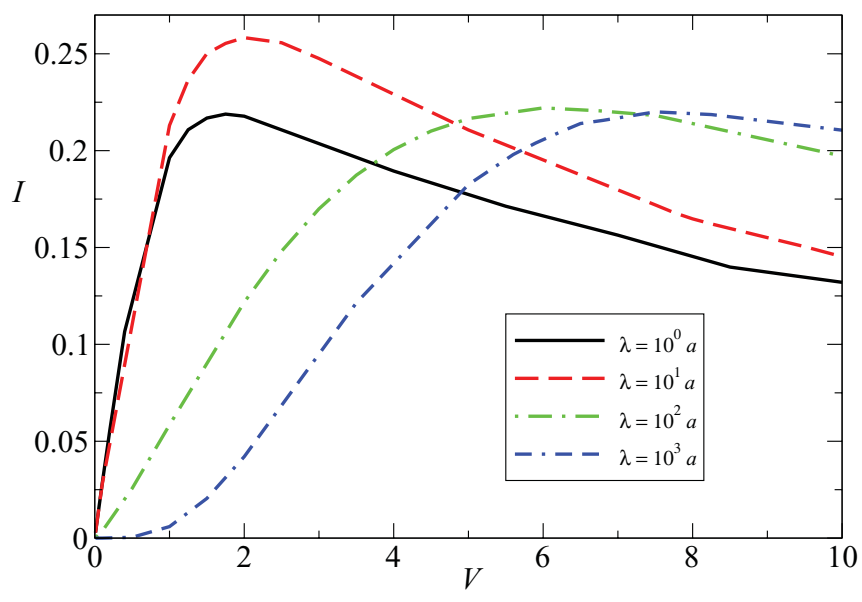

FIG. 4. (Color online) $I-V$ curve for a soft-Yukawa dot-lead interaction $U(x)=W e^{-|x| / \lambda} / \sqrt{x^{2}+a^{2}}$ with $W=0.15$ and for different values of $\lambda$. $W$ is in units of $2 \pi v$. The other of parameters are like in Fig. 2. 


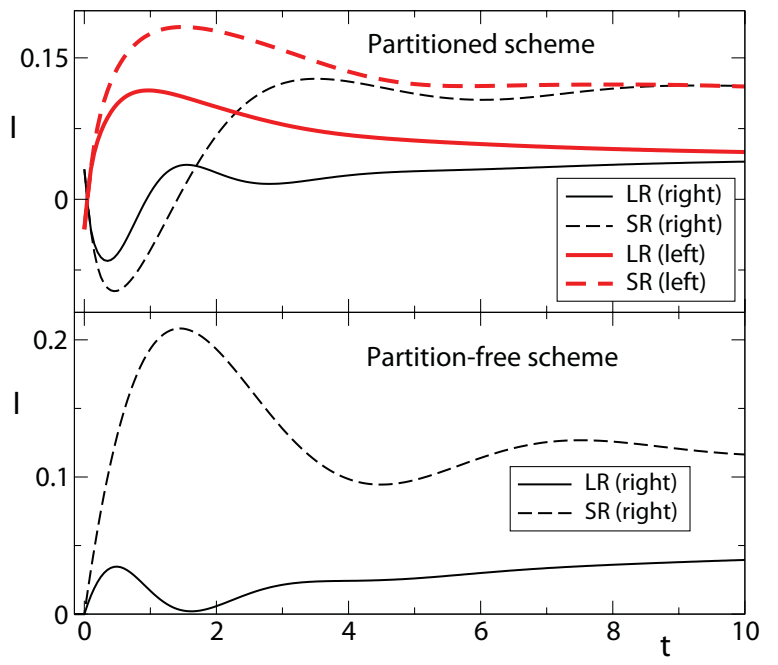

FIG. 5. (Color online) Time-dependent current for SR (dashed) and LR (solid) interaction for an initial density $n=0$. The thick curves represent $I_{L}(t)$ while the thin curves $I_{R}(t)$. The parameters are $V_{L}=-V_{R}=1, \gamma=0.2, U=W=0.2, \bar{\varepsilon}_{d}=0$. In the top panel $t_{0}=0$ (partitioned scheme) while in the bottom panel $t_{0}=60$ (simulated partition-free scheme). Units are $10^{-1} v / a$ for $V_{\alpha}, \gamma, \tilde{\varepsilon}_{d}$, $10 a / v$ for $t$ and $2 \pi v$ for $U$ and $W$.

dot-lead interaction was neglected. In the 2D quantum Hall liquid the current through the dot is carried by the edge state electrons which, unscreened by the bulk electrons, experience a long-range interaction with the charged dot. At present there is no experimental evidence that in these systems the zero-bias conductance is $\sigma_{0}$ at resonance. In fact, in Ref. 36 values below $\sigma_{0}$ were reported.
LR interactions have an impact also in the screening time. In Fig. 5 we plot the time-dependent currents for LR and SR interaction with same interaction strength $W=U .{ }^{37}$ The LR current relaxes faster both in the partitioned scheme (contacts and bias switched on simultaneously at $t=0$ ) and partition-free scheme. The same behavior is observed for different values of $W$ (not shown). The jamming effect of LR interactions is at the origin of the faster screening time: electrons respond to a change in the dot population suddenly and well deep inside the leads.

Finally we observe that the steady-state value of the current is the same in both schemes. This agrees with the results of Refs. 23-26 according to which the memory of the initial state is washed out in the long-time limit.

\section{CONCLUSION}

In conclusion we presented a comprehensive characterization of the transport properties of the IRLM with LR interactions. We proposed an embedding scheme based on a suitable truncation of the EOM for the dressed fermion fields and derived KBEs, which we solved numerically and benchmarked against available exact results. Even though we explicitly considered a single-level dot, no complications arise with more complex systems. The method was compared with recently proposed RE approaches, and found to be superior from the transient (no negative densities) to the steady-state regime (no severe underestimation of the dot polarizability). LR interactions leave clear fingerprints in the time-dependent current as well as in the $I-V$ curve. In particular, we predict a suppression of the zero-bias conductance when the level is in resonance with the lead Fermi energy.
${ }^{1}$ D. V. Averin and K. K. Likharev, Mesoscopic Phenomena in Solids (Elsevier, Amsterdam, 1991).

${ }^{2}$ A. C. Hewson, The Kondo Problem to Heavy Fermions (Cambridge University Press, Cambridge, 1993).

${ }^{3}$ H. Grabert and M. H. Devoret, Single Charge Tunneling: Coulomb Blockade Phenomena in Nanostructures (Plenum, New York, 1992).

${ }^{4}$ J. B. Neaton, M. S. Hybertsen, and S. G. Louie, Phys. Rev. Lett. 97, 216405 (2006).

${ }^{5}$ K. S. Thygesen and A. Rubio, Phys. Rev. Lett. 102, 046802 (2009).

${ }^{6}$ M. Strange, C. Rostgaard, H. Häkkinen, and K. S. Thygesen, Phys. Rev. B 83, 115108 (2011).

${ }^{7}$ Changsheng Li, M. Bescond, and M. Lannoo, Phys. Rev. B 80, 195318 (2009).

${ }^{8}$ P. Myöhänen, R. Tuovinen, T. Korhonen, G. Stefanucci, and R. van Leeuwen, Phys. Rev. B 85, 075105 (2012).

${ }^{9}$ E. Boulat, H. Saleur, and P. Schmitteckert, Phys. Rev. Lett. 101, 140601 (2008).

${ }^{10}$ A. Nishino, T. Imamura, and N. Hatano, Phys. Rev. Lett. 102, 146803 (2009).

${ }^{11}$ C. Karrasch, M. Pletyukhov, L. Borda, and V. Meden, Phys. Rev. B 81, 125122 (2010).
${ }^{12}$ C. X Sheng, M. Tong, and Z. V. Vardeny, Phys. Rev. B 81, 205103 (2010).

${ }^{13}$ J. Spitaler, E. Y. Sherman, H. G. Evertz, and C. Ambrosch-Draxl, Phys. Rev. B 70, 125107 (2007).

${ }^{14}$ D. Bohr and P. Schmitteckert, Phys. Rev. B 75, 241103 R (2004).

${ }^{15}$ H. Ness and L. K. Dash, Phys. Rev. B 84, 235428 (2011).

${ }^{16}$ Y. Meir and N. S. Wingreen, Phys. Rev. Lett. 68, 2512 (1992).

${ }^{17}$ A.-P. Jauho, N. S. Wingreen, and Y. Meir, Phys. Rev. B 50, 5528 (1994).

${ }^{18}$ F. Elste, D. R. Reichman, and A. J. Millis, Phys. Rev. B 83, 245405 (2011).

${ }^{19}$ R. S. Whitney, J. Phys. A: Math. Theor. 41, 175304 (2008).

${ }^{20} \mathrm{~T}$. Giamarchi, Quantum Physics in One Dimension (Clarendon, Oxford, 2004).

${ }^{21}$ J. Gonzàlez, M. A. Martín-Delgado, G. Sierra, and M. A. H. Vozmediano, Quantum Electron Liquids and High- $T_{c}$ Superconductivity (Springer-Verlag, Berlin, 1995).

${ }^{22}$ M. Cini, Phys. Rev. B 22, 5887 (1980).

${ }^{23}$ G. Stefanucci and C. O. Almbladh, Phys. Rev. B 69, 195318 (2004).

${ }^{24}$ P. Myöhänen, A. Stan, G. Stefanucci, and R. van Leeuwen, Europhys. Lett. 84, 67001 (2008).

${ }^{25}$ E. Perfetto, G. Stefanucci, and M. Cini, Phys. Rev. Lett. 105, 156802 (2010). 
${ }^{26}$ V. Moldoveanu, H. D. Cornean, and C.-A. Pillet, Phys. Rev. B 84, 075464 (2011).

${ }^{27}$ The term $\frac{U_{0}}{\mathcal{L}} \frac{1}{i}\left\langle\mathcal{T}\left\{\hat{N}_{\alpha}(z) d(z) d^{\dagger}\left(z^{\prime}\right)\right\}\right\rangle$ renormalizes the energy $\tilde{\varepsilon}_{d}$. Indeed $\left.\left.N_{\alpha} / \mathcal{L} 0\right\rangle=\hat{\rho}_{\alpha} 0\right\rangle$ with $\hat{\rho}_{\alpha}$ the equilibrium density of lead $\alpha$. Since the dot induces fluctuations of or$\operatorname{der} O(1 / \mathcal{L})$ we have $\frac{U_{0}}{\mathcal{L}} \frac{1}{i}\left\langle\mathcal{T}\left\{\hat{N}_{\alpha}(z) d(z) d^{\dagger}\left(z^{\prime}\right)\right\}\right\rangle=U_{0} \hat{\rho}_{\alpha} G\left(z, z^{\prime}\right)+$ $O(1 / \mathcal{L})$.

${ }^{28}$ R. van Leeuwen, N. E. Dahlen, G. Stefanucci, C. O. Almbladh, and U. von Barth, Time-Dependent Density Functional Theory (Springer, New York, 2006); Lect. Notes Phys. 706, 33 (2006); M. Cini, Topics and Methods in Condensed Matter Theory (Springer-Verlag, Berlin, 2007).

${ }^{29}$ N. E. Dahlen and R. van Leeuwen, Phys. Rev. Lett. 98, 153004 (2007).
${ }^{30}$ P. Myöhänen, A. Stan, G. Stefanucci, and R. van Leeuwen, Phys. Rev. B 80, 115107 (2009).

${ }^{31}$ M. Puig von Friesen, C. Verdozzi, and C.-O. Almbladh, Phys. Rev. B 82, 155108 (2010).

${ }^{32}$ K. Balzer, S. Bauch, and M. Bonitz, Phys. Rev. A 82, 033427 (2010).

${ }^{33} \Sigma^{\mathrm{R}}(\omega) \propto \gamma \ll v / a$ is purely imaginary and slowly varying in $\omega$.

${ }^{34}$ P. Mehta and N. Andrei, Phys. Rev. Lett. 96, 216802 (2006).

${ }^{35}$ A. Furusaki, Phys. Rev. B 57, 7141 (1998).

${ }^{36}$ O. M. Auslaender, A. Yacoby, R. de Picciotto, K. W. Baldwin, L. N. Pfeiffer, and K. W. West, Phys. Rev. Lett. 84, 1764 (2000).

${ }^{37}$ This comparison is based on the idea of having an interaction $U_{q}=$ $U\left[(1-\mu)-\mu \ln (a q)^{2}\right]$ parametrically dependent on $\mu . U_{q}$ is SR for $\mu=0$ whereas is LR for $\mu=1$. 